Pressekonferenz

\title{
Ultraschall: ein wichtiges Instrument bei der Früherkennung und Diagnostik von Brustkrebs
}

Über Fortschritte und neue Techniken der Ultraschalldiagnostik zur Früherkennung und Behandlung von Brustkrebs informierten Ultraschall-Experten am Donnerstag, den 21. März 2013 auf der Pressekonferenz der Deutschen Gesellschaft für Ultraschall in der Medizin (DEGUM) in den Räumen der Bundespressekonferenz Berlin. Die anwesenden Medienvertreter, darunter Journalisten des WDR-Hörfunks, des Berliner Kuriers, der Ärzte Zeitung und des Deutsches Ärzteblatts diskutierten mit den Experten über den Sinn von Ultraschalluntersuchungen, die das Mammografie-Screening ergänzen, über die Bedeutung einer hohen Gewebedichte der Brust als Risikofaktor für Brustkrebs und über den „Ultraschall der Zukunft“.

Referenten und Ansprechpartner für die Medien waren Professor Dr. med. Dr. h.c. Friedrich Degenhardt (Leiter des Arbeitskreises Mammasonografie der DEGUM, Chefarzt der Klinik für Frauenheilkunde und Geburtshilfe, Franziskus Hospital, Bielefeld und Leiter des Brust-zentrums Bielefeld-Herford), Professor Dr. med.
Jens-Uwe Blohmer (Chefarzt der Abteilung Frauenheilkunde und Geburtshilfe, Leiter des Brustzentrum-City, Sankt Gertrauden-Krankenhaus, Berlin), Dr. med. Volker Duda (Oberarzt und Leiter des Arbeitsbereiches „Senologische Diagnostik“ an der Klinik für Gynäkologie, gynäkologische Endokrinologie und Onkologie am

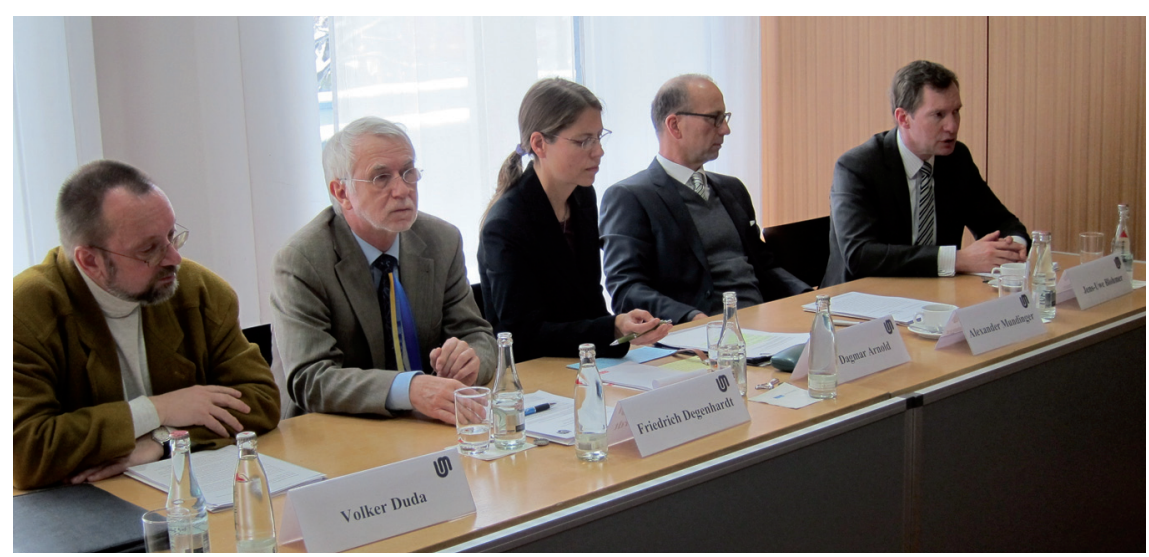

Universitätsklinikum Gießen und Marburg) und Professor Dr. med. Alexander Mundinger (Chefarzt der Klinik für Radiologie, Marienhospital Osnabrück, Chefarzt der Sektion Bildgebende und minimalinvasive Mammadiagnostik des Brustzentrums am Franziskus-Hospital Harderberg, Direktor des Zentrums für Radiologie der Niels-Stensen-Kliniken, Osnabrück).

Neueste Datenauswertungen zeigen, dass der zweijährige Rhythmus der Röntgenuntersuchung im Rahmen des Mammografie-Screening-Programms zur Brustkrebsfrüherkennung bei manchen Frauen nicht ausreicht: So ergab die Datenauswertung von fast 880000 Screening-Teilnehmerinnen in Nordrhein-Westfalen: Bei über 2000 als unauffällig eingestuften Frauen wurde noch vor der nächsten regulären Mammografie Brustkrebs entdeckt. Häufig seien Frauen mit einer erhöhten Gewebedichte der Brust von diesen schnellwachsendenden „Intervallkarzinomen“ betroffen, berichteten die Experten auf der Pressekonferenz.

Das neue Österreichische Screeningverfahren sieht für diese Risikopatientinnen eine Kombination von Mammografieund Ultraschall-Screening - im jährlichen Wechsel vor. In Deutschland sei dies aufgrund der knappen Ressourcen derzeit 
nicht möglich, erklärten die Referenten. Jede Frau habe jedoch die Möglichkeit, die Ultraschalluntersuchung der Brust als IGeL-Leistung in Anspruch zu nehmen. Die Mediziner legten den anwesenden Journalisten dar, für welche Patientinnen die Untersuchung empfehlenswert ist und worauf sie bei der Suche nach der geeigneten Praxis achten sollten. Entgegen der weitläufigen Meinung, nach der nur jüngere Frauen ein „dichtes Brustgewebe“ aufwiesen, gäbe es auch ältere Frauen mit diesem Merkmal. Zudem könnten auch jüngere Frauen, die noch nicht am Mammografie-Screening teilnehmen, die - risikofreie - Ultraschalluntersuchung machen lassen. Entscheidend sei es dabei, sich eine Einrichtung mit einem leistungsstarken Ultraschallgerät zu suchen. Für ein verlässliches Ergebnis sollten die Ultraschallköpfe eine Schallfrequenz von zehn bis 15 Megahertz aufweisen.

Zudem sprachen die Experten mit den Medienvertretern über neueste technolo- gische Entwicklung im Bereich der Mammasonografie. Die Zukunft gehöre automatisierten 3-D-Techniken wie der „automatisierte Volumendarstellung der Brust ${ }^{\text {“ }}$ (ABVS). Diese Technik ermögliche eine lückenlose Untersuchung und Dokumentation des gesamten Brustgewebes. Aufgrund der hohen Anschaffungskosten des Gerätes, die „in der Größenordnung eines Einfamilienhauses“ lägen, und der bislang noch nicht ausreichend vorhandenen wissenschaftlichen Daten findet das Verfahren in Deutschland bislang noch keine breite Anwendung. Mit einer zunehmenden Anzahl wissenschaftlich fundierter Studien würde auch die Zahl der Anwender steigen, zeigten sich die Experten überzeugt. Bereits heute gehörten die verschiedenen Techniken der Mammasonografie zur täglichen Praxis bei der Diagnostik von Brustkrebs sowohl als eigenständige Methode als auch in Ergänzung zur Röntgendiagnostik und Magnetresonanztomografie. 